\title{
Clinical Tip: Extraction of a difficult to remove intramedullary antibiotic rod in the ankle
}

\author{
by Ronald Belczyk, DPM ${ }^{1} \rrbracket$, Dane K. Wukich, MD $^{2} \square$
}

The Foot \& Ankle Journal 1 (7): 6

The purpose of this clinical tip is to describe a technique which can facilitate extraction of an antibiotic impregnated polymethylmethacrylate (PMMA) spacer from the intramedullary canal of the hindfoot, ankle and distal tibia. This method employs the Revision Moreland instrumentation system which was originally designed for total hip revision surgery.

Key words: Antibiotic rod, antibiotic spacers, bone cement, Moreland extractor

Published: July 2008

This is an Open Access article distributed under the terms of the Creative Commons Attribution License. It permits unrestricted use, distribution, and reproduction in any medium, provided the original work is properly cited. (The Foot \& Ankle Journal (www.faoj.org)

$\mathbf{T}_{\mathrm{h}}$ he use of polymethylmethacrylate antibiotic spacers is commonly used in the management of osteomyelitis of the hip, knee, and ankle joint. This method permits a local delivery of concentrated antibiotics while avoiding elevated systemic levels. ${ }^{1} \quad$ After removal of internal hardware and debridement of necrotic tissue and/or bone, the dead space needs to be managed. Spacers made of antibiotic impregnated cement were designed to prevent soft tissue contraction and maintain extremity alignment while awaiting prosthetic implantation or arthrodesis type procedures.
Typically, these spacers are shaped according to the size and shape of the defect. In the ankle joint, intramedullary spacers are constructed in the shape of a cylinder with the use of a ball tipped pin, polymethylmethacrylate (PMMA) cement, and heat stabile antibiotics.

It may be a challenge to remove the intramedullary spacer in some patients. Several methods and instrumentation have been described for the extraction of bone cement in revision hip and knee arthroplasties. ${ }^{2}$ Dr. Moreland designed instrumentation to facilitate revisional hip and knee surgery. The Moreland revision instruments contain cement extractors which aid in the removal of previously placed prosthesis and bone cement.

Address correspondence to: Dane Wukich, MD. UPMC

Comprehensive Foot and Ankle Center. Roesch-Taylor Bldg Ste 7300.

2100 Jane St. Pittsburgh, PA 15203. Phone: 412-586-1546 Fax: 412-

586-1544

Email: wukichdk@upmc.edu

\footnotetext{
${ }^{1}$ PGY-4, Fellow, Foot and Ankle Surgery, University of Pittsburgh Medical Center, Pittsburgh, Pennsylvania, 15203.

${ }^{2}$ Chief, Foot and Ankle Division, University of Pittsburgh Medical Center Department of Orthopedic Surgery and Assistant Professor, University of Pittsburgh School of Medicine, Pittsburgh, Pennsylvania, 15203.
} 


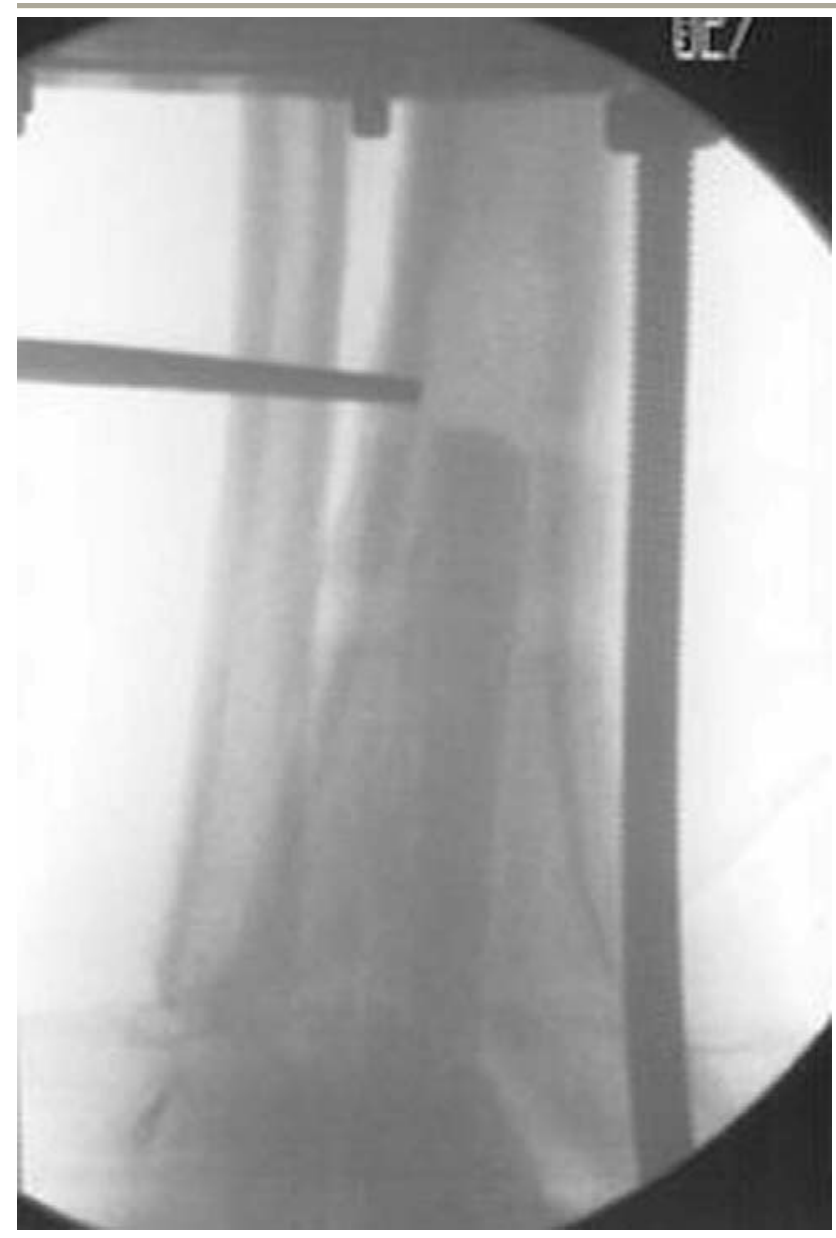

Figure 1 AP radiograph demonstrates an intramedullary placed antibiotic impregnated polymethylmetacrylate rod in the distal tibia.

\section{Description of Technique}

The patient is placed in the supine position and an incision is made on the plantar aspect of the foot. If the antibiotic rod cannot be extracted easily from the inferior aspect of the calcaneus, a cement extractor from the revision Moreland system is then obtained and inserted in a retrograde fashion. If necessary, a small window can be made in the tibia proximal to the antibiotic rod or at the level of the ankle joint to grasp the intramedullary spacer with bone forceps. (Fig.1) This will prevent proximal migration of the antibiotic rod during extraction. The extractor is then inserted into the canal from the plantar entry site until the triangular hook lies proximal to the rod.

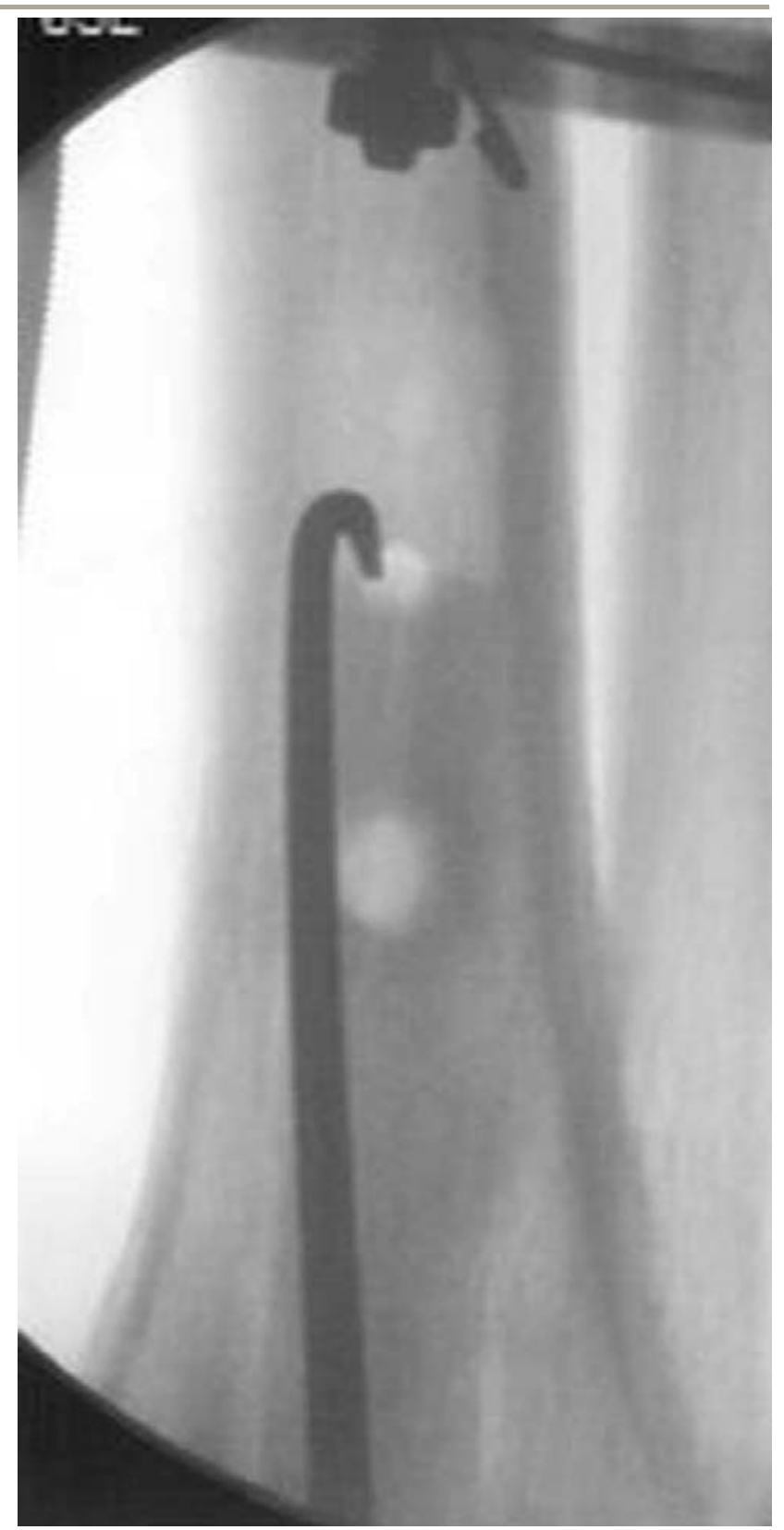

Figure 2 Extraction of the broken intramedullary antibiotic rod with the use of a Moreland revision extractor.

C-arm visualization helps to ensure proper placement and prevent proximal migration of the rod.

The hook is then rotated in a clockwise or counterclockwise manner to gain purchase on the superior edge of the spacer. (Fig. 2) 


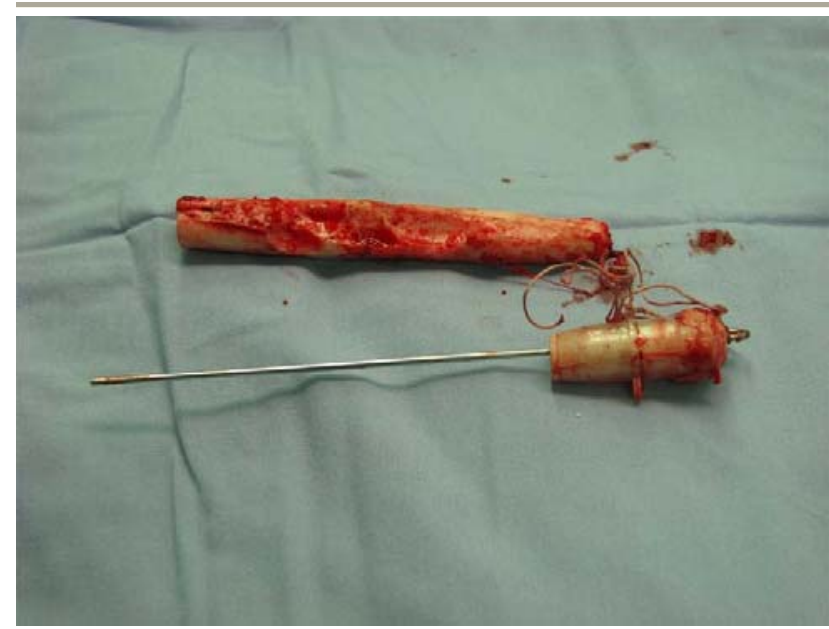

Figure 3 Intra-operative pictures of a broken antibiotic rod.

A slap hammer is used to extract the antibiotic rod in a anterograde fashion. Once the rod is removed proper joint debridement and biopsy is obtained. (Fig. 3)

At the time of implantation of an intramedullary spacer we also recommend placing a bulb tipped guidewire or a long Steinman pin that it is hooked at the proximal end through the middle of the polymethylmethacrylate spacer. The distal end of guidewire or Steinman should be left proud in the soft tissues to facilitate extraction.

In summary, removal of cemented rods may be a challenge to the operating foot and ankle surgeon. However, the revision Moreland instrumentation can be useful. (Fig. 4) Several companies have cement removal hand tools with reverse hooks which are similar in design to the one reported in this study. Another method of cement removal not discussed here is with ultrasonically driven cement removal instruments. ${ }^{3-6}$ They are used to assist with the removal of poylmethylmethacrylate (PMMA) bone cement during arthroplasty revision. The potential advantages of using the hand tools versus the ultrasonic instrumentation is the avoidance of cell death at the endosteal bone surface, hazardous fumes from the melting of the PMMA bone cement, and soft tissue damage from heat or friction burns.

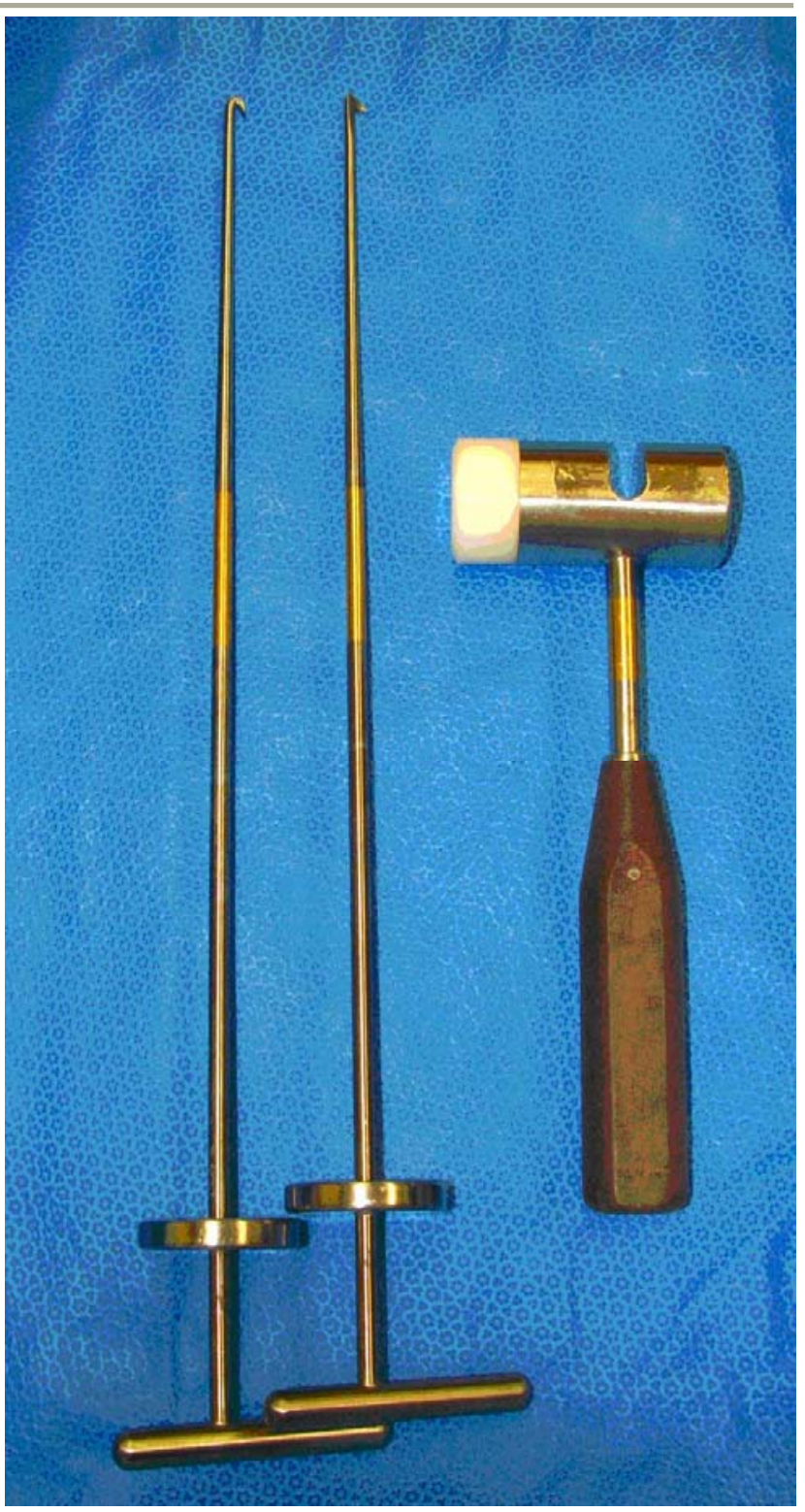

Figure 4 Curved hook extractors and slotted mallet found in the Revision Moreland instrumentation system. 


\section{References}

1. Springer, B.L., G; Osmon,D., Systemic Safety of High Dose Antibiotic Loaded Cement Spacers after Resection of an Infected Total Knee Arthroplasty. Clinical Orthopaedics and Related Research, 427: p. 47-51, 2004.

2. Moreland, J.M., R; Anspach,W., The Window technique for the removal of broken femoral stems in total hip replacement. Clinical Orthopaedics and Related Research, 212: p. 245-249, 1986.

3. Fletcher, M., Jennings, G.J., Warren, P.J. Ultrasonically driven instruments in the transfemoral approach-an aid to preservation of bone stock. and reduction of implant length Arch Othop Trauma Surg, 120: p. 559561, 2000.

4. Smith, P., Eyres, K. Safe removal of massive intrapelvic cement using ultrasonic instruments J Arthroplasty, 14: p. 235-238, 1999.

5. Callaghan, J., Elder, S., Stranne, S., Fulghum, C., Seaber, A., Myers, B. Revision arthroplasty facilitated by ultrasonic tool cement removal. An evaluation of whole bone strength in a canine model J Arthroplasty, 7: p.495-500, 1992.

6. Klapper, R., Caillouette, J., The use of ultrasonic tools in revision arthroplasty procedures Contemp Orthop, 20: 273-279, 1990. 